\title{
DNA methylation studies in plants based on sequencing technologies
}

\author{
P. Arrigo ${ }^{1 *}$, A.A. Anashkina ${ }^{3}$, N.G. Esipova ${ }^{3}$, O.B. Dobrovolskaya ${ }^{4,5}$, Y.L. Orlov ${ }^{4,5 *}$ \\ ${ }^{1}$ ISMAC Institute, Genova, Italy \\ ${ }^{3}$ Engelhardt Instittue of Molecular Cell Biology RAS, Moscow, Russia \\ ${ }^{4}$ I nstitute of Cytology and Genetics SB RAS, Novosibirsk, Russia \\ ${ }^{5}$ Novosibirsk State University, Novosibirsk, Russia \\ *e-mail: orlov@bionet.nsc.ru
}

Key words: epigenetics, methylation, plants, next-generation sequencing, nucleosome, ncRNA

Motivation and Aim: The epigenetic mechanisms regulating phenotype expression could be studied now using novel sequencing technologies. Based on the commonly accepted definition, this term refers to all phenotypic variation that does not require a nucleotide change in the DNA. The epigenetic regulation varies from molecular mechanism such as DNA methylation to high order modification such as nucleosome positioning o chromosome unfolding. In the last decade it is emerged the critical role of epigenetic modification of long term exposure to environmental stress factors and stress response in plants. In an environmental sustainability perspective, the alteration of epigenetic regulation can also impact biodiversity.

Methods and Algorithms: The harmful effect of pollutants on human epigenetic control is largely demonstrated, however the effects on plants and, more generally on a specific ecological community, requires more studies. Several plants such as Arabidopsis, are became a system model to study different molecular mechanisms. The high complexity of plants genome organization requires to dedicate more studies in order to tackle the different epigenetic regulation in these biological systems. This kind of research has great impact in order to optimize the crop production under the pressure of climate change and pollution. The new high-throughput molecular techniques have allowed to enhance the capability to investigate the events that affect the epigenetic regulation. We guess that a particular relevance can be assigned to the chemical modifications of informational macromolecules (DNA and RNA) that could be considered as starting point of epigenetic regulation.

Results and conclusion: The molecular investigation of epigenetic process is greatly supported extensively by immunoprecipitation sequencing (ChIP-seq) and methylome microarray. These high-throughput techniques permit to maps a large number of methylated sites on DNA and to evaluate their role in transcriptional processes. The long non coding RNAs seem to be involved in the control of trasncriptional process [1]. It is also important to underline that ncRNA are also capable to contribute to nucleosome positioning. We will review current state of art in this area in the frames of new joint collaborative project.

Acknowledgements: The research has been supported by RFBR No. 18-54-00037. Computing done at Siberian Supercomputer center SB RAS was supported by budget project No. 0324-2018-0017.

\section{References}

1. Wang J., Meng X., Dobrovolskaya O.B., Orlov Y.L., Chen M. (2017) Non-coding RNAs and their roles in stress response in plants. Genomics Proteomics Bioinformatics. 15(5):301-312. 